\title{
Silicon Photonics for Microwave Photonics Applications
}

\author{
Lawrence R. Chen, Senior Member, IEEE, Fellow, OSA
}

\author{
(Invited Paper)
}

\begin{abstract}
We provide an overview of recent work on developing integrated microwave photonic subsystems in silicon photonics for generating chirped microwave waveforms as well as for providing optical true time delay. First, we describe onchip spectral shapers based on Bragg gratings (BGs), including a distributed Fabry-Pérot cavity, a Michelson interferometer incorporating identical chirped BGs, and a Sagnac loop incorporating a chirped BGs, for use in wavelength-to-time mapping systems. The performance of these on-chip spectral shapers is compared to those based on microring resonators as well as fiber or free-space configurations. Second, we consider the development of an index variable optical true time delay line (OTTDL) using subwavelength grating (SWG) waveguides. For SWG waveguides of the same length, incremental delays can be obtained by tailoring the group index via control over the duty cycle. We compare the performance of SWG waveguide OTTDLs to other length variable implementations in silicon.
\end{abstract}

Index Terms-Silicon photonics, microwave photonics, Bragg gratings, subwavelength grating waveguides, optical true time delay, photonic generation and processing of microwave signals

\section{INTRODUCTION}

$\mathrm{M}^{\mathrm{i}}$ icrowave photonics (MWP) unites the disciplines of microwave and optical engineering and focuses on the use of photonic techniques and technologies to generate, process, and analyze/characterize microwave signals [1-4]. By exploiting the capabilities of photonics for providing broad bandwidth, parallelization, and adaptiveness (e.g., to achieve readily reconfiguration and tuning), the value added of MWP is that it supports the realization of a number of important functions in microwave systems that are either too complex or not possible to implement with conventional electronic approaches.

Applications of MWP have evolved significantly since the early idea of using optical means to transport/distribute microwave signals over long distances and include, amongst others, broadband communications, sensing, and instrumentation. In support of these applications, a number of functions are required, including photonic generation of arbitrary waveforms, e.g., microwave, millimeter wave, and

Manuscript received May 31, 2016. This work was supported in part by the Natural Sciences and Engineering Research Council of Canada.

L. R. Chen is with the Department of Electrical and Computer Engineering, McGill University, Montreal, QC H3A 0E9 Canada (email: lawrence.chen@mcgill.ca).
THz signals; photonic processing of microwave signals, e.g., filtering, time delay, and phase shifting; and photonic characterization of microwave signals, e.g., spectrum analysis and instantaneous frequency measurement (IFM).

In recent years, significant efforts have been directed on developing integrated photonic technologies to realize microwave photonic signal processing functions [5-10]. This has been motivated by the need to address outstanding issues of 'bulk' MWP systems and MWP signal processing engines, particularly the lack of compactness, stability, and reliability, as well as performance. To date, the main MWP integration platforms considered are silica, silicon nitride, silicon-oninsulator (SOI), InP, $\mathrm{LiN}_{\mathrm{b}} \mathrm{O}_{3}$, and chalcogenide glasses (see [6] for a review). Each presents advantages and/or strengths in terms of passive optical signal routing and processing, integration with (RF) electronics, light generation, and photodetection. Indeed, highly sophisticated (sub-)systems in InP [5], silicon nitride [7], and SOI [10] have been realized; moreover, nonlinear integrated microwave photonic circuits in chalcogenide to implement highly selective filters or to perform broadband IFM have been demonstrated [9].

In this paper, we discuss recent work on silicon photonics for microwave arbitrary waveform generation, such as chirped microwave waveforms, and microwave signal processing, specifically the implementation of broadband optical true time delay lines (OTTDLs). The remainder of this paper is organized as follows. In Section 2, we describe the photonic generation of chirped microwave waveforms based on spectral shaping and wavelength-to-time mapping (WTM). We review the development of on-chip spectral shapers based on waveguide Bragg gratings (BGs) and provide a comparison of such spectral shapers to those based on ring resonators and other fiber or free-space approaches. In Section 3, we present the use of subwavelength grating (SWG) waveguides as a means to realize index variable OTTDLs. The performance of these OTTDLs is compared to that of conventional length or wavelength variable optical delay lines (ODLs). Finally, conclusions and an outlook are given in Section 4.

\section{Generation of Chirped Microwave And Millimeter WAVE WAVEFORMS}

\section{A. General Overview}

Microwave and millimeter wave waveforms are needed in broadband (wireless) communications as well as imaging and 
impulse radar. In particular, chirped microwave and millimeter wave waveforms can be compressed in order to improve measurement resolution and increase detection distance in radar systems [11]. Characteristics of the waveforms include the central frequency, RF chirp, time bandwidth product [a large TBWP is needed to achieve a high pulse compression ratio], stability (e.g., pulse to pulse uniformity), and signal-tonoir ratio. We are particularly interested in the first three.

Photonic generation of microwave and millimeter wave waveforms can overcome limitations associated with electronic means, particularly with digital-to-analog converters. Over the years, many approaches for photonic generation of microwave and millimeter wave waveforms have been explored. The use of optical pulse shaping to synthesize a desired optical (temporal) waveform followed by photodetection is highly popular since it provides flexibility in terms of reconfiguration and tunability and indeed, a wealth of optical pulse shaping techniques are available [12]. In terms of ease of implementation, optical spectral shaping followed by WTM is very attractive [13-18]. A spectral shaper is used to tailor the amplitude spectrum of a pulsed broadband optical source and the shaped spectrum then propagates through a dispersive medium where the frequency content is distributed in the time domain, i.e., WTM, see Fig. 1.

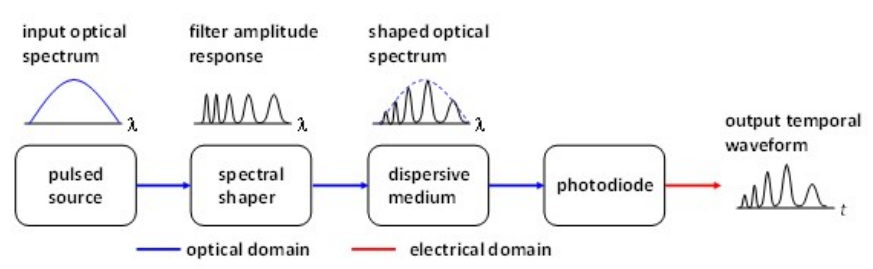

Fig. 1. Principle for photonic generation of chirped microwave waveforms based on optical spectral shaping and WTM.

Generating a uniform microwave waveform (constant frequency or chirp-free) requires a spectral shaper with a periodic filter response, i.e., constant free spectral range (FSR), and a linear WTM. To obtain a chirped waveform, two approaches can be adopted: (1) the use a spectral shaper with a constant FSR followed by a nonlinear WTM (e.g., [17]) or alternatively, (2) the use of a spectral shaper with a variable FSR followed by a linear WTM. In this paper, we focus on the latter, which avoids the lack of readily available integrated dispersive media with nonlinear delay. The key element in these waveform generation systems is the spectral shaper: it must allow for the synthesis of the desired amplitude spectrum which will ultimately correspond to the desired waveform after WTM.

In this section, we review different on-chip spectral shapers based on BGs, in particular the distributed Fabry-Pérot cavity (DFPC), a Michelson interferometer incorporating chirped Bragg gratings, and a Sagnac loop incorporating a chirped Bragg grating. We then compare the ability of these on-chip spectral shapers to generate chirped microwave waveforms with other integrated approaches such as ring resonators as well as fiber and free-space implementations.
A number of different techniques exist to characterize the generated chirped waveforms, including the use of a highbandwidth photodiode and sampling oscilloscope (or a realtime oscilloscope) to visualize the time-domain waveform or an electrical (RF) spectrum analyzer for spectrum analysis. With an RF spectrum analyzer, only the power spectrum is typically available. On the other hand, the variation in the instantaneous frequency as a function of time can be obtained from the time-domain waveform through post processing, e.g., calculations using the Hilbert transform or spectrogram distributions. The results presented below are based on post processing calculations.

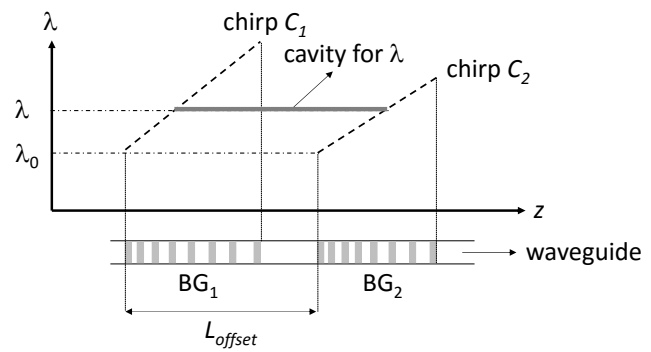

Fig. 2. Schematic of a spectral shaper based on a DFPC comprising two spatially offset linearly chirped BGs.

\section{B. Distributed Fabry-Pérot cavity}

A DFPC can be created using (linearly) chirped BGs that are spatially separate or overlapping (partially or fully) as shown schematically in Fig. 2 [19-21]. Such a DFPC has an FSR that is wavelength dependent [21]:

$$
\operatorname{FSR}(\lambda)=\frac{\lambda_{0}^{2}}{2 n_{\text {eff }} L(\lambda)}=\frac{\lambda_{0}^{2}}{2 n_{\text {eff }}\left(L_{\text {offset }}+\frac{C_{1}-C_{2}}{C_{1} C_{2}}\left(\lambda-\lambda_{0}\right)\right)}
$$

where $L(\lambda)$ represents the wavelength dependent, equivalent cavity length; $n_{\text {eff }}$ is the effective index of the waveguide, $C_{l}$ and $C_{2}$ are the linear chirps of the gratings (in $\mathrm{nm} / \mathrm{mm}$ or $\mathrm{nm} / \mathrm{cm}), L_{\text {offset }}$ is the spatial offset between the gratings measured from the start of each grating, and $\lambda_{0}$ is the starting wavelength. After WTM in a dispersive medium with a firstorder dispersion coefficient $\ddot{\Phi}_{\lambda}$ (in units of ps/nm), the instantaneous frequency of the microwave waveform can be approximated by

$$
f_{R F}(t) \propto 2 n_{e f f}\left[\frac{C_{1}-C_{2}}{C_{1} C_{2}} \times\left(\frac{t}{\lambda_{0}^{2} \ddot{\Phi}_{\lambda}^{2}}-\frac{1}{\lambda_{0} \ddot{\Phi}_{\lambda}}\right)+\frac{L_{\text {offset }}}{\lambda_{0}^{2} \ddot{\Phi}_{\lambda}}\right]
$$

and the corresponding RF chirp is

$$
\frac{d f_{R F}(t)}{d t}=2 n_{e f f}\left(\frac{C_{1}-C_{2}}{C_{1} C_{2}}\right) \frac{1}{\lambda_{0}^{2} \ddot{\Phi}_{\lambda}^{2}}
$$

We consider a fixed dispersive medium providing a fixed WTM. If the grating chirps are fixed, the central frequency $f_{R F}$ 
of the waveform (i.e., at $t=0$ ) is determined by the amount of spatial offset $L_{\text {offset }}$ between the two gratings. On the other hand, the RF chirp is independent of $L_{\text {offset }}$ and depends only on the grating chirps.

Wang and Yao demonstrated an all-fiber DFPC based on two spatially separate identical linearly chirped fiber Bragg gratings with opposite orientation (i.e., $L_{\text {offset }}>L_{g}$ where $L_{g}$ is the length of the BG and $C_{1}=-C_{2}$ ) [21]. The use of identical gratings simplifies fabrication and as shown in (2) and (3), opposite orientations (or chirp rates) enables higher values of central frequency and RF chirp. With an all-fiber DFPC based on $10 \mathrm{~mm}$ long linearly chirped fiber Bragg gratings having identical grating chirps $\left|C_{l}\right|=\left|C_{2}\right|=C=0.1 \mathrm{~nm} / \mathrm{mm}$ and $L_{\text {offset }}=12 \mathrm{~mm}$, linearly chirped microwave waveforms with $f_{c}$ $=15 \mathrm{GHz}$, an RF chirp $=0.0217 \mathrm{GHz} / \mathrm{ps}$, and a TBWP $=37.5$ (with corresponding pulse compression ratio of 62.5) were generated using $58 \mathrm{~km}$ of single mode fiber (SMF) for WTM.

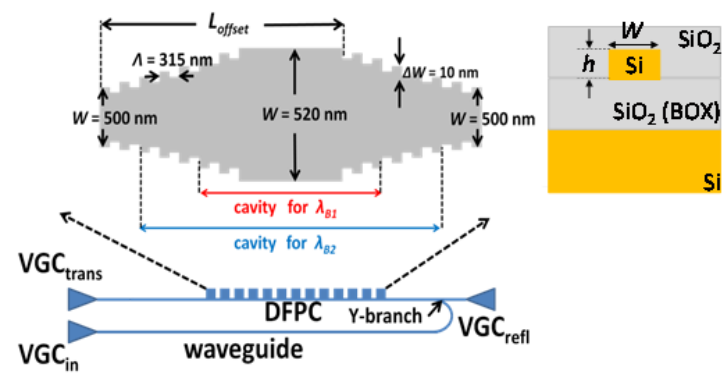

Fig. 3. Schematic of the on-chip DFPC spectral shaper in SOI along with the waveguide cross-section.

We implemented the DFPC design in SOI using parameters and processes typical of silicon photonic multi-project wafer runs (our devices were fabricated at the University of Washington Nanofabrication Facility using electron beam lithography with a single full etch) [22]. The DFPC (see Fig. 3 ) employs silicon nanowire waveguides with a cross-section of $h \times W=220 \times 500 \mathrm{~nm}$ (the waveguides support the fundamental TE-like mode only); they sit on a $3 \mu \mathrm{m}$ buried oxide (BOX) layer and have a $2 \mu \mathrm{m}$ thick index-matched top oxide cladding. As with the all-fiber DFPC demonstrated in [21], we use two identical linearly chirped BGs with opposite orientation that are separated spatially by a length $L_{\text {offset }}$. Of the different possible approaches to realize BGs, we have opted to use sidewall corrugations with a corrugation depth $\Delta W$ directly in the nanowire waveguides [23-24]. The chirp is obtained by using a constant grating period and tapering the waveguide width [25], which is more robust compared to varying the grating period. Each $B G$ has a grating period $\Lambda=$ $315 \mathrm{~nm}$, a corrugation depth $\Delta W=10 \mathrm{~nm}$, and 500 periods (the corresponding grating length is $L_{g}=157.5 \mu \mathrm{m}$ ). The waveguide width $W$ increases linearly from $500 \mathrm{~nm}$ to $520 \mathrm{~nm}$ (or from $520 \mathrm{~nm}$ to $500 \mathrm{~nm}$ ) from one end of the grating to the other and the estimated grating chirp is $\left|C_{l}\right|=\left|C_{2}\right|=C \sim$ $12.2 \mathrm{~nm} / \mathrm{mm}$.

For spectral shaping, we use the reflection response of the DFPC, which is extracted via a Y-branch splitter [23]. Vertical grating couplers (VGCs) are used for input and output coupling and are optimized for TE mode operation [23]. The total fiber-to-fiber insertion loss is $25 \mathrm{~dB}$ with each VGC contributing $\sim 10 \mathrm{~dB}$ loss. The total size of the on-chip spectral shaper is $720 \mu \mathrm{m} \times 70 \mu \mathrm{m}$.

Fig. 4 shows the measured reflection spectra of two on-chip DFPCs with $L_{\text {offset }}=307.5 \mu \mathrm{m}$ and $457.5 \mu \mathrm{m}$. As expected from (1), the FSR at a specific wavelength is smaller when $L_{\text {offset }}$ is larger and in both cases, the FSR increases with detuning from $\lambda_{0}$ (note that since we use $C_{1}>0$, the equivalent cavity length decreases with increasing detuning from the starting wavelength $\lambda_{0}$ so that there is a corresponding increase in FSR).
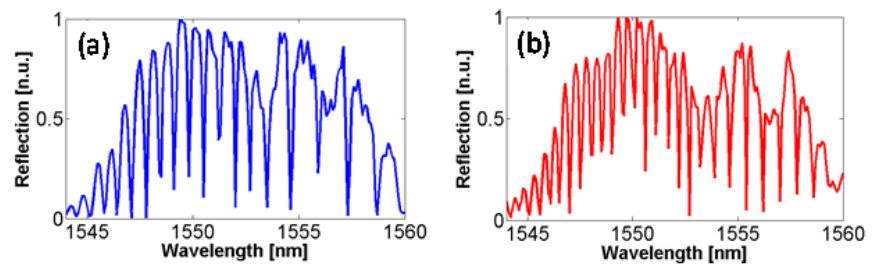

Fig. 4. Reflection spectra of on-chip DFPC spectral shaper in SOI with $L_{\text {offset }}=$ $307.5 \mu \mathrm{m}$ (a) and $457.5 \mu \mathrm{m}$ (b).

To generate chirped microwave waveforms, we use a pulsed broadband source (femtosecond laser) and $5 \mathrm{~km}$ of SMF for WTM (an optical bandpass filter is used after the laser so that the broadband source spectrum matches the reflection bandwidth of the DFPCs). The results are summarized in Fig. 5: the values of $f_{R F}$ and $\mathrm{RF}$ chirp are $15 \mathrm{GHz}$ and 0.011 $\mathrm{GHz} / \mathrm{ps}$, and $20 \mathrm{GHz}$ and $0.012 \mathrm{GH} / \mathrm{ps}$ for $L_{\text {offset }}=307.5 \mu \mathrm{m}$ and $457.5 \mu \mathrm{m}$, respectively. As predicted by (2) and (3), increasing $L_{\text {offset }}$ yields higher values of $f_{R F}$ without changing the RF chirp. The generated waveforms are $\sim 1,100$ ps in duration and the estimated TBWPs are 13.3 and 14.5. The compressed pulses, which are obtained by calculating the autocorrelation of the measured waveforms, have full width at half maximum (FWHM) durations of 120 ps and $50 \mathrm{ps,}$ corresponding to pulse compression ratios of 9 and 12 .
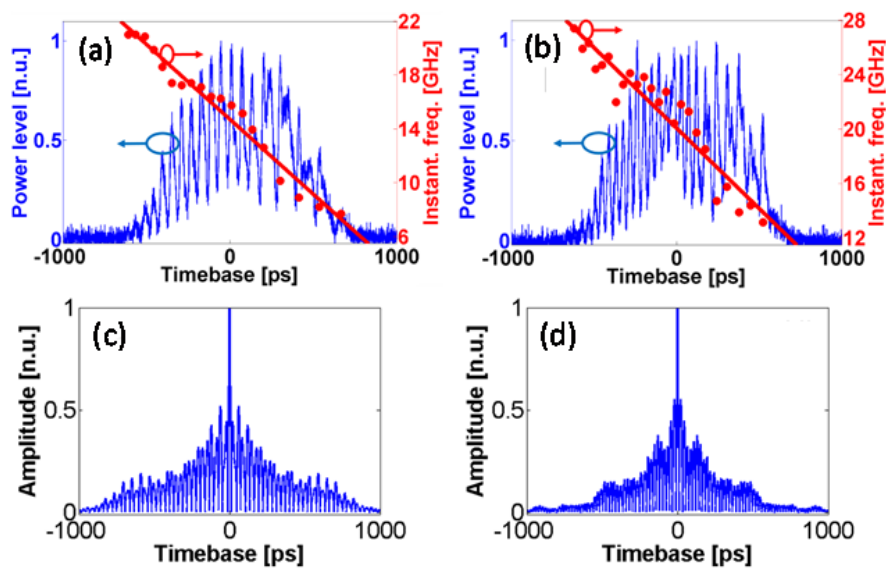

Fig. 5. Generating chirped microwave waveforms using on-chip DFPC spectral shaper in SOI. Temporal waveforms and instantaneous frequency vs. time $(\mathrm{a}, \mathrm{b})$ and calculated auto-correlation showing pulse compression $(\mathrm{c}, \mathrm{d})$ with $L_{\text {offset }}=307.5 \mu \mathrm{m}$ (left) $457.5 \mu \mathrm{m}$ (right). 


\section{Michelson Interferometer with Chirped Bragg Gratings}

A simple means to create a filter with a periodic spectral response is to use a $3 \mathrm{~dB}$ coupler and two identical uniform period BGs to form a Michelson interferometer, see Fig. 6(a) [26]. An offset placed in one branch of the interferometer enables control of the interference of light being reflected back from the two gratings (and hence, the FSR of the resulting filter). To create an aperiodic spectral response, chirped BGs can be used. If this filter is used as a spectral shaper, the expressions for the wavelength dependent FSR, $f_{R F}$, and RF chirp of the generated waveforms are also given by (1) - (3) with $L_{\text {offset }}$ corresponding to the wavelength independent path difference created by the physical offset of the BGs in the interferometer arms and $\lambda_{0}$ being the center, rather than starting, wavelength (here, $L(\lambda)$ represents the length difference between the arms of the interferometer and includes the wavelength independent path difference/offset as well as the wavelength dependent length difference due to the grating chirp).

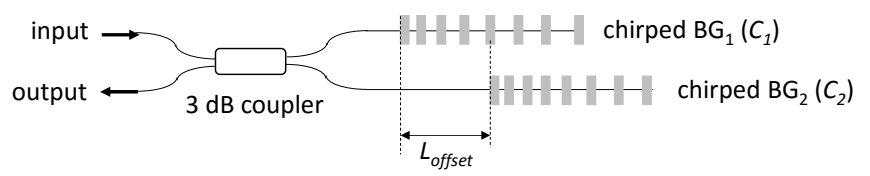

(a)
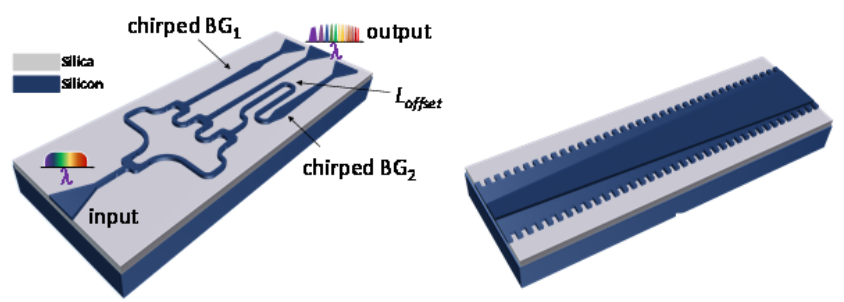

(b)

Fig. 6. Spectral shaper based on a Michelson interferometer incorporating two chirped BGs: (a) general schematic and (b) on-chip implementation in SOI with chirped BGs having opposite orientation and perspective view of chirped BGs (courtesy of J. Yao).

In contrast to the DFPC, it is easier to implement a wider range of values of $L_{\text {offset }}$ with the Michelson interferometer. While fully or partially superimposed fiber Bragg gratings can be fabricated readily to have $L_{\text {offset }}<L_{g}$ (due to the physical mechanism of photoinduced refractive index changes), it is more difficult to realize the corresponding 'superimposed' grating profiles with on-chip BGs based on sidewall corrugations. For $L_{\text {offset }}=0$, the spectral response is symmetric about $\lambda_{0}$ and the FSR decreases with increasing detuning (i.e., the largest value of FSR is obtained at $\lambda_{0}$ ). For a sufficiently large offset, the spectral response is no longer symmetric and there is a monotonic increase or decrease in FSR.

Zhang and Yao recently demonstrated an on-chip version of the Michelson interferometer incorporating oppositely chirped gratings in SOI as shown in Fig. 6(b) [27]. Silicon nanowire waveguides are used for all passive interconnections and the Y-branches. On the other hand, a rib waveguide is used for the BGs. The gratings use sidewall corrugations on the slab and the chirp is obtained by tapering the rib width. The BGs have a length $L_{g}=12.54 \mathrm{~mm}$, a nominal chirp of $0.88 \mathrm{~nm} / \mathrm{mm}$, and span a wavelength range from $1538 \mathrm{~nm}$ to $1544 \mathrm{~nm}$. Using a length of dispersion compensating fiber with a dispersion of $-1700 \mathrm{ps} / \mathrm{nm}$ for WTM, the corresponding generated chirped microwave waveforms for two spectral shapers with $L_{\text {offset }}=0$ and $L_{\text {offset }}=L_{g}$ are shown in Fig. 7. For $L_{\text {offset }}=0$, the spectral response is symmetric about the center wavelength whereas the FSR increases monotonically with wavelength when $L_{\text {offset }}=L_{g}$. For the latter device, the generated chirped microwave waveform has $f_{R F}=15.4 \mathrm{GHz}$, an $\mathrm{RF}$ chirp $=0.00154 \mathrm{GHz} / \mathrm{ps}$, and a TBWP $=615$ (with a pulse compression ratio as high as 623 ).

\section{Sagnac Loop}

One possible drawback of the spectral shaper based on the Michelson interferometer is the need for two identical BGs. Instead, a single grating can be used in a Sagnac interferometer as illustrated in Fig. 8 [28]. The wavelength independent offset is controlled by the path mismatch, i.e., $L_{\text {offset }}=L_{1}-L_{2}$. With a single chirped grating, the Sagnac loop provides the same functionality as the Michelson interferometer with two identical and oppositely chirped BGs. As such, the wavelength dependent FSR, $f_{R F}$, and RF chirp are again given by $(1)-(3)[L(\lambda)$ has the same interpretation as in the case of the Michelson interferometer].

Wang and Yao developed an all-fiber version of this spectral shaper using a $10 \mathrm{~mm}$ long linearly chirped fiber Bragg grating with a grating chirp $C=2 \mathrm{~nm} / \mathrm{cm}$ [29]. They included a tunable ODL in one path to vary $L_{\text {offset }}$ (e.g., to have either a monotonic increase or decrease in FSR). As a proofof-principle demonstration, they used $30.8 \mathrm{~km}$ of SMF for WTM and generated linearly chirped microwave waveforms with $f_{R F}=20.2 \mathrm{GHz}(24.5 \mathrm{GHz}), \mathrm{RF}$ chirps of $0.02 \mathrm{GHz} / \mathrm{ps}(-$ $0.022 \mathrm{GHz} / \mathrm{ps}$ ), and TBWP $=44.8(41)$ with corresponding pulse compression ratios of 54.7 (56.5).

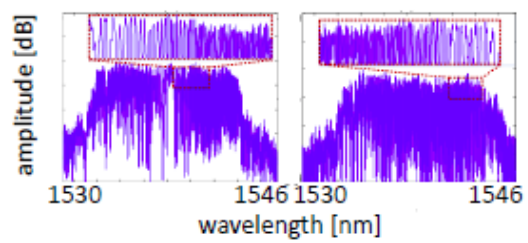

(a)
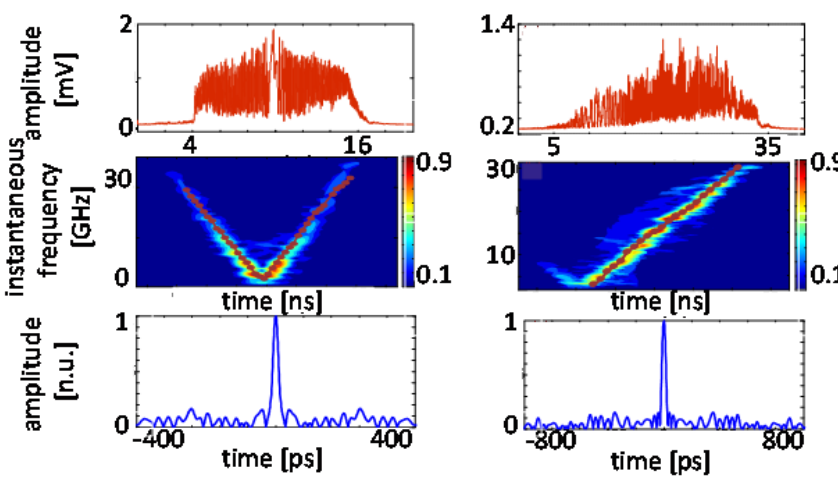

(b)

Fig. 7. Generating chirped microwave waveforms using on-chip spectral shaper based on a Michelson interferometer incorporating two oppositely chirped BGs in SOI. (a) Spectral response, (b) temporal waveform (top), instantaneous frequency (middle), and calculated compressed pulse (bottom) for $L_{\text {offset }}=0$ (left) and $L_{g}$ (right) (courtesy of J. Yao). 


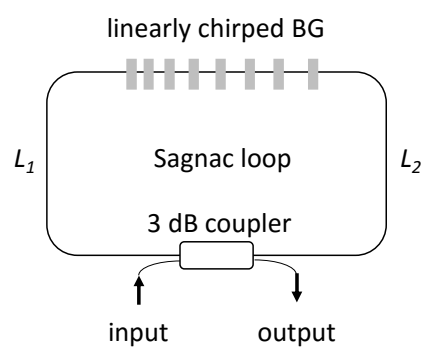

Fig. 8. Schematic of a spectral shaper based on a Sagnac loop incorporating a linearly chirped BG.

We have implemented the Sagnac loop based spectral shaper in SOI using the same platform and fabrication processes as with the on-chip DFPCs [30]. As before, we use nanowire waveguides with a cross-section of $h \times W=220 \mathrm{~nm}$ $\times 500 \mathrm{~nm}$. $3 \mathrm{~dB}$ coupling is provided by a multi-mode interference (MMI) coupler; it is $6 \mu \mathrm{m}$ wide by $127 \mu \mathrm{m}$ long and the input and output nanowire waveguides are separated by $3 \mu \mathrm{m}$ to avoid additional coupling. The BG has a uniform period $(\Lambda=320 \mathrm{~nm})$ and sidewall corrugations $\Delta W=10 \mathrm{~nm}$; the chirp is obtained by varying linearly the waveguide width from $500 \mathrm{~nm}$ to $510 \mathrm{~nm}$ from one end of the grating to the other (the estimated grating chirp is $C \sim 0.26 \mathrm{~nm} / \mathrm{mm}$ ). The grating comprises 10,000 periods for a total length $L_{g}=3.2$ $\mathrm{mm}$. Due to the different waveguide widths at one end of the chirped grating and the input/output of the MMI coupler, we employ a $10 \mu \mathrm{m}$ long taper to bring the waveguide width at the wide end of the grating from $510 \mathrm{~nm}$ back to $500 \mathrm{~nm}$.

Fig. 9 shows the spectral responses for two on-chip Sagnac loop spectral shapers with $L_{\text {offset }}=100 \mu \mathrm{m}$ and $400 \mu \mathrm{m}$. These values of path mismatch are insufficient to induce a monotonic increase or decrease in FSR over the grating bandwidth $(\sim 12$ $\mathrm{nm})$, i.e., the spectral response is still somewhat symmetric about the center wavelength $\lambda_{0}$ and the FSR decreases with increasing detuning away from $\lambda_{0}$. However, by tuning the center wavelength of a pulsed broadband source with a bandwidth that is smaller than the grating bandwidth, we can generate chirped microwave waveforms having different $f_{R F}$ and/or RF chirp of opposite sign.

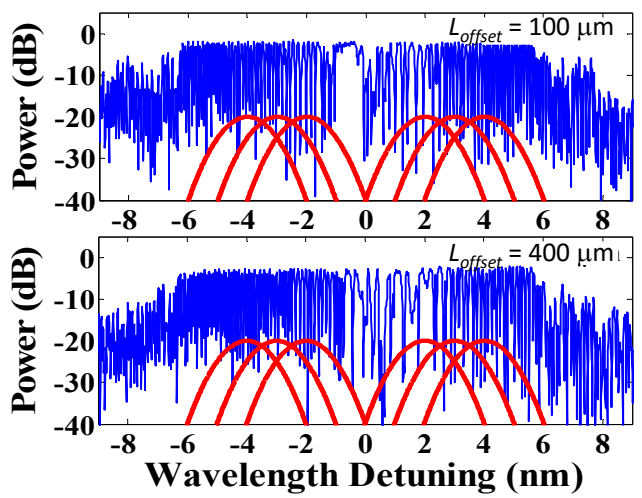

Fig. 9. Spectral responses of on-chip spectral shaper based on a Sagnac loop incorporating a chirped BG with $L_{\text {offset }}=100 \mu \mathrm{m}$ (a) and $400 \mu \mathrm{m}$ (b). The normalized spectra of the broadband pulses used for generating chirped microwave waveforms are also shown (red).

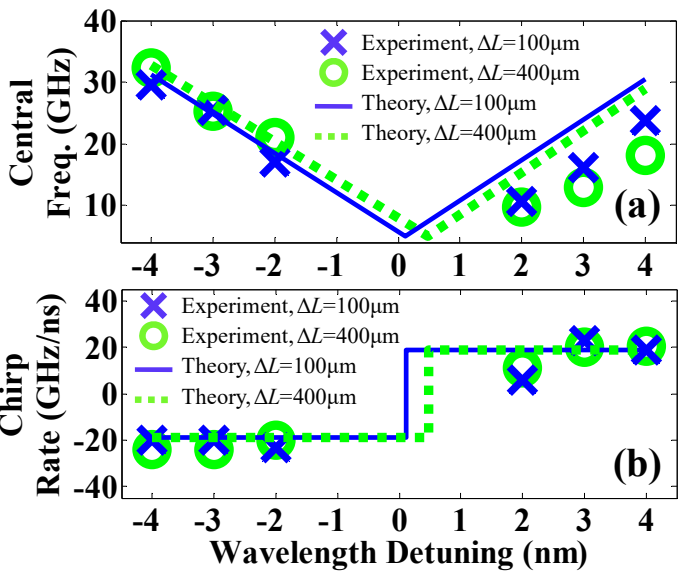

Fig. 10. Characteristics of chirped microwave waveform generated using an on-chip spectral shaper based on a Sagnac loop incorporating a chirped BG. (a) $f_{R F}$ and (b) RF chirp as a function of detuning between the center wavelength of the pulsed broadband source and the spectral shaper response (for the measured points, the corresponding overlap between the pulsed broadband source and spectral shaper response are shown in Fig. 9).

As a demonstration, we use an optical bandpass filter to reduce the FWHM bandwidth of our pulsed broadband source to $1.5 \mathrm{~nm}$ and tune its center wavelength to various portions of the grating response as shown in Fig. 9; we also use $24 \mathrm{~km}$ of SMF for WTM. The results are summarized in Fig. 10: a central frequency of up to $30 \mathrm{GHz}$ can be obtained with an RF chirp of $\sim \pm 0.02 \mathrm{GHz} /$ ps. Fig. 11 illustrates typical chirped microwave waveforms and the TBWPs are between 15 and 20.
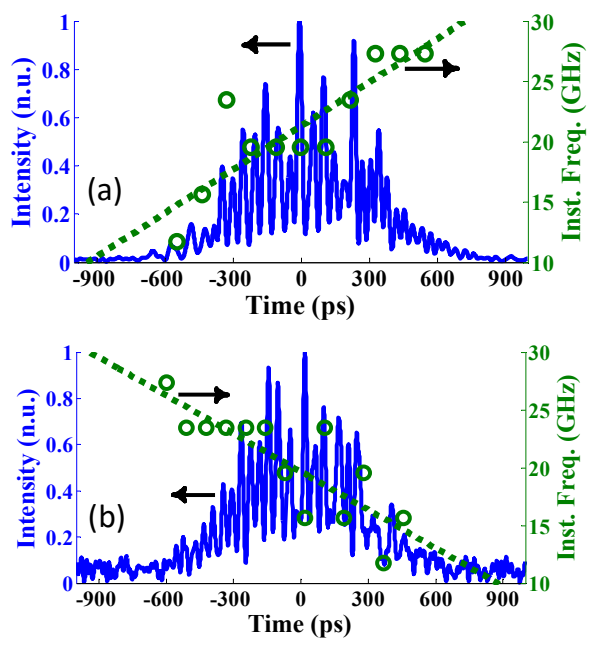

Fig. 11. Temporal waveform and instantaneous frequency vs. time for chirped microwave waveform generated using an on-chip spectral shaper based on a Sagnac loop incorporating a chirped BG with $L_{\text {offset }}=100 \mu \mathrm{m}$ and a detuning of $-4 \mathrm{~nm}$ (a) and $4 \mathrm{~nm}$ (b) between the central wavelength of the pulsed broadband source and the spectral shaper response.

\section{E. Discussion}

Table 1 compares the on-chip spectral shapers based on BGs in SOI for generating chirped microwave waveforms, particularly with respect to RF chirp and TBWP. Results from all-fiber implementations, where available, are also included. The TBWP depends on the grating chirp $C$ and the bandwidth 
of the spectral shaper (denoted $\left.B_{\lambda}\right)[27,29]$ :

$T B W P \propto \frac{B_{\lambda}^{2}}{C}$

or equivalently,

$$
T B W P \propto C L_{g}
$$

since $B_{\lambda}$ is directly proportional to $C$ and $L_{g}$. Achieving a higher TBWP requires an increase in the grating chirp $C$ and/or the grating length $L_{g}$ (note that $B_{\lambda}$ is constrained by the requirement for a linear WTM, e.g., it must be constrained so that higher-order dispersion in the dispersive medium used to implement WTM can be neglected). For the on-chip spectral shaper based on a Sagnac loop incorporating a chirped BG described in Section 2D, a maximum TBWP of 233 is possible based on the chirped grating characteristics. However, in the experiments, since the bandwidth of the pulsed broadband optical source was restricted to $1.5 \mathrm{~nm}$ to provide tunability, only a fraction of the spectral shaper bandwidth was used $\left(B_{\lambda}\right.$ $=1.5 \mathrm{~nm}$ ), giving rise to the demonstrated TBWPs of $15-20$ only. While longer grating lengths are more readily available in fiber compared to waveguides in SOI, the TBWP of on-chip configurations can provide greater TBWPs compared to their all-fiber counterparts.

TABLE I

COMPARISON OF RF CHIRP AND TBWP OF CHIRPED MICROWAVE WAVEFORMS GENERATED USING ON-CHIP AND ALL-FIBER SPECTRAL SHAPERS BASED ON BGS AND MRRS

\begin{tabular}{|l|l|c|c|c|c|}
\hline \multirow{2}{*}{} & & \multicolumn{2}{|c|}{ On-chip in SOI } & \multicolumn{2}{c|}{ All-fiber } \\
\cline { 3 - 6 } & $\begin{array}{l}\text { RF chirp } \\
(\mathrm{GHz} / \mathrm{ps})\end{array}$ & TBWP & $\begin{array}{c}\text { RF chirp } \\
(\mathrm{GHz} / \mathrm{ps})\end{array}$ & TBWP \\
\hline \hline \multirow{3}{*}{ BG } & DFPC & 0.011 & $9-12$ & 0.0217 & 38 \\
& Michelson & 0.0154 & 615 & N/A & N/A \\
\cline { 2 - 7 } & Sagnac & 0.02 & $15-20$ & 0.02 & 45 \\
\hline \multirow{3}{*}{ MRR } & $\begin{array}{l}\text { Conventional } \\
\text { add/drop }\end{array}$ & 0.011 & 6.5 & N/A & N/A \\
\cline { 2 - 7 } & MZI & 0.017 & $\sim 20$ & N/A & N/A \\
\hline
\end{tabular}

In addition to BGs, on-chip spectral shapers with an aperiodic filter response (i.e., variable FSR) can be realized by cascading multiple MRRs having different resonant wavelengths. For example, Khan et al. cascaded 8 MRRs, each with an FSR of $\sim 16 \mathrm{~nm}$ and a wavelength separation of $\sim 1.3 \mathrm{~nm}$ between consecutive rings, in a conventional add/drop configuration in SOI [31]. Within the $16 \mathrm{~nm}$ FSR, 8 wavelengths were 'dropped' from a pulsed broadband optical source (corresponding to 8 time domain 'cycles' after WTM); chirped microwave waveforms with $f_{R F} \sim 10 \mathrm{GHz}$ and an RF chirp $\sim 0.01 \mathrm{GHz} / \mathrm{ps}$ were obtained. As a second example, Zhang and Yao used 5 cascaded MRRs in a Mach-Zehnder interferometer (MZI) to generate chirped microwave waveforms with $f_{R F}=15.5 \mathrm{GHz}$ and an $\mathrm{RF}$ chip $=0.017$ $\mathrm{GHz} / \mathrm{ps}$ [10]. One advantage of this configuration is that the input and output share a common coupling port which will increase robustness and tolerance to fabrication errors, especially when VGCs are used. While MRRs are suitable for high-density integration and support greater compactness compared to BGs, the limited number of MRRs that can be cascaded will, in turn, limit the achievable TBWP.

Other passive optical pulse shaping techniques as well as active systems have been investigated for generating chirped microwave and millimeter wave waveforms. Of particular note are interferometric spectral shapers which have been implemented in-fiber [32] or with free space optics [33]. With the latter, Rashidinejad and Weiner generated chirped microwave waveforms spanning the $70-110 \mathrm{GHz}$ frequency range with a TBWP as large as 600 for high resolution Wband ranging applications [34]. Additionally, chirped waveforms with a record-high TBWP of up to 70,000 have been realized by heterodyne beating of a continuous wave and wavelength-swept laser (or pre-chirped pulse) [35].

While on-chip spectral shapers may not match the performance of active systems, they offer several interesting features. First, by introducing a pn junction to the silicon waveguide and applying a bias voltage, reconfiguration can be enabled through the plasma dispersion effect. In particular, if the junction is introduced along the rib waveguide $\mathrm{BG}$, the grating chirp, and hence RF chirp and TBWP, can be tuned. Indeed, this approach was adopted by Zhang and Yao to realize electrical tuning of the on-chip spectral shaper based on a Michelson interferometer incorporating chirped BGs; the generated chirped microwave waveforms had TBWPs ranging from 95 to 143 [36]. On the other hand, tuning the wavelength independent offset $L_{\text {offset }}$ may be possible if the junction is introduced in the offset waveguide; this will allow for control over $f_{R F}$.

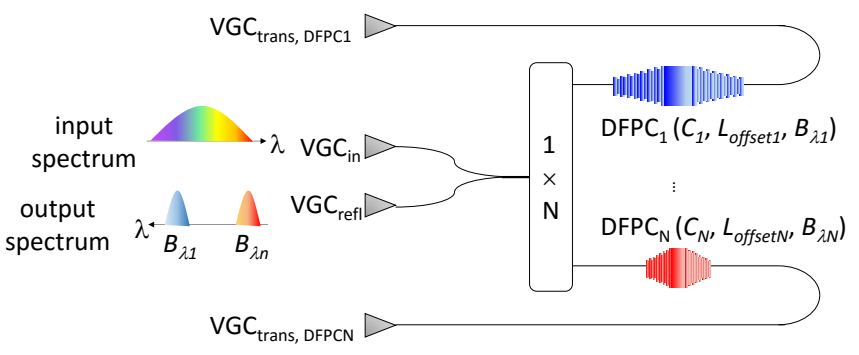

(a)

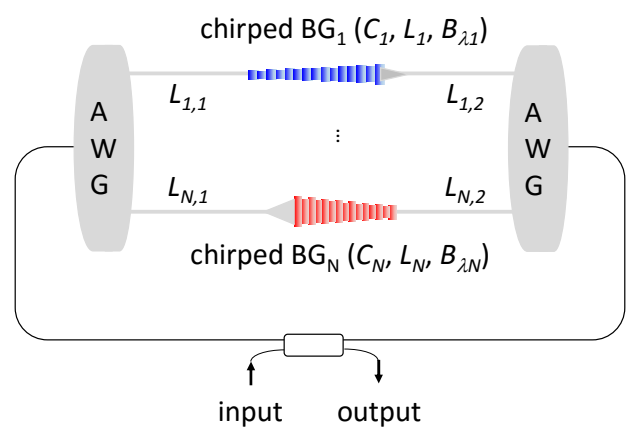

(b)

Fig. 12. Design concept for multi-carrier chirped waveforms using on-chip spectral shapers based on (a) parallel DFPCs and (b) arrayed waveguide Sagnac interferometer incorporating chirped BGs. 
Second, we can exploit the parallelism of optics to generate multiple chirped microwave waveforms with different characteristics ( $f_{R F}$ and RF chirp) simultaneously using a single device structure. For example, as shown in Fig. 12(a), DFPCs can be designed to occupy separate spectral bands and arranged in parallel to generate wavelength-divisionmultiplexed (WDM) waveforms. Recently, high-performance arrayed waveguide gratings (AWGs) in SOI have been realized $[37,38]$. Thus, a second alternative to obtain WDM chirped waveforms is to incorporate chirped BGs in an arrayed waveguide Sagnac interferometer [39]. This latter implementation exhibits greater flexibility for reconfiguration via tuning the BG or $L_{\text {offset }}$. The WDM nature of the generated waveforms may provide enhanced capabilities for radar, ranging, and instrumentation applications.

\section{Optical True-Time Delay Lines}

\section{A. General Overview}

An ODL is a fundamental building block that enables numerous signal processing functions in MWP systems and applications [40,41]. For example, they are used to develop MWP filters and devices to control phase shift. ODLs are also important for beamforming in phased array antennas, especially to avoid beam squint which can arise when using electronic delay lines [42-45]. Characteristics of ODLs include, amongst others, large delay, small incremental delay steps (especially for beamforming as the operating frequency of the antenna system increases) or continuous delay, broad operating bandwidth, and low loss. Note that the specific features that an ODL is required to provide are applicationsspecific and not all are required simultaneously.

There are a number of ways to implement an optical delay passively, i.e., without gain, and these can be broken down in two broad approaches: (1) variation of the propagation length $(L)$ of the delay element (i.e., the delay $\Delta t$ is given by $L / v_{\mathrm{g}}$ where $v_{g}$ is the propagation group velocity), also known as a length variable delay line [43-49] and (2) variation of the propagation group velocity $\left(v_{\mathrm{g}}\right)$, also referred to as a variable propagation velocity line and for some implementations, a wavelength variable delay line [50-59]. These two approaches encompass employing a fixed length of waveguide, fiber, or free space as the delay medium, as well as resonance enhancements, whereby the physical length of the delay medium is enhanced, e.g., through a cavity or exploiting a material resonance where dispersion can be large. The use of resonance enhancements is appealing and indeed, optical filters based on cascaded MRRs, which are all-pass filters having large delay on resonance and a unity magnitude response, have been used to develop continuously tunable delays (see, e.g., [7,51-53]). However, there is a well-known trade-off between the amount of resonance enhancement that can be obtained and the operating bandwidth [60]. In this paper, we focus on ODLs that do not involve resonance enhancements. Integrated ODLs are more compact, provide greater temporal resolution, and greater stability compared to fiber or micro-electro-mechanical-systems.

A wavelength variable ODL can be implemented using a highly dispersive waveguide to create group velocity variation among different wavelengths $(\lambda)$, see Fig. 13(a). In this case, optical pulses or signals need to be carried on different wavelengths (or carrier frequencies), e.g., $\lambda_{1}, \lambda_{2}$, etc., to experience different propagation velocities and correspondingly, different propagation time delays, see Fig. 13(d). Integrated wavelength variable ODLs have been demonstrated using photonic crystal waveguides and Bragg gratings. For example, in SOI, a variable delay of several hundred picoseconds over an optical bandwidth of a few nm's can be obtained with chirped waveguide BGs [25] while a (nonlinear) delay of up to $70 \mathrm{ps}$ over $20 \mathrm{~nm}$ was obtained with a photonic crystal waveguide [57]. While such ODLs are important as sampled delay lines for MWP filters, they cannot be used in applications where the time delays must be obtained at the same wavelength.

(a) wavelength-variable ODL
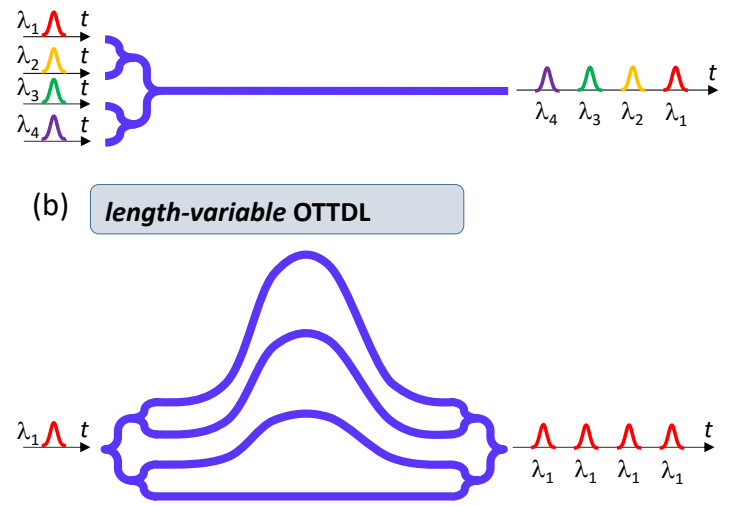

(c) index-variable OTTDL

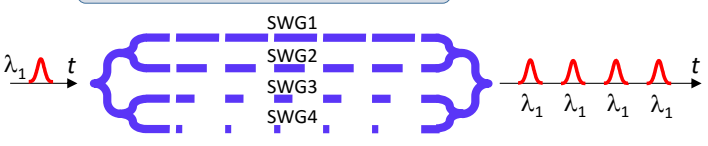

(d)

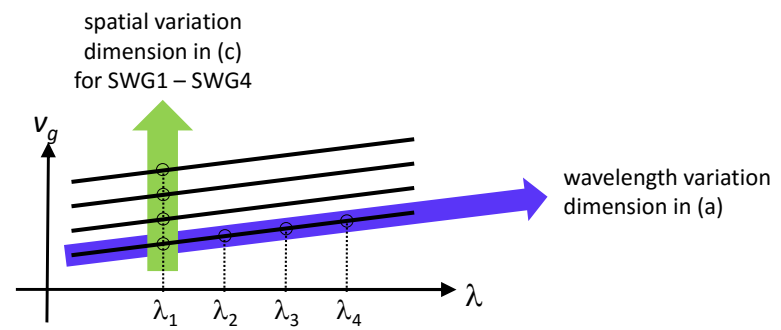

Fig. 13. Illustration of the conventional approach for wavelength-variable ODL (a), the conventional approach for length-variable OTTDL (b), and our proposed approach of index-variable OTTDL (c). Two comparisons between these approaches are illustrated in the plot of $v_{\mathrm{g}} \mathrm{vs} . \lambda$ in (d).

An ODL providing time delays for pulses/signals having the same optical carrier/wavelength is one form of an optical true time delay line (OTTDL). The conventional architecture of such a length variable OTTDL is depicted in Fig. 13(b) where the (incremental) time delay between adjacent waveguides (also referred to as taps) is obtained by changing the length of each waveguide. Note that for (discretely) tunable OTTDLs, the different lengths of waveguides are commonly interconnected via $2 \times 2$ or $1 \times N / N \times 1$ switches 
(or splitters/combiners). To minimize chip size, such delay architectures generally involve waveguides with curvy or spiral/serpentine topologies. Impressive results have been demonstrated in both SOI and silicon nitride. For example, a 7 bit (discretely tunable) delay with a maximum delay of 1.27 ns delay and $10 \mathrm{ps}$ resolution (step size) based on 8 cascaded 2 $\times 2$ MZI switches and 7 waveguide paths in SOI was reported [49]. The minimum delay path in each stage of the device was $4 \mathrm{~mm}$ and incremental path differences in integer multiples of $808 \mu \mathrm{m}$ were used. Ridge waveguides with a wide waveguide width of $3 \mu \mathrm{m}$ were considered to reduce sidewall scattering loss The waveguides were arranged in a serpentine manner and the total chip size was $7.4 \mathrm{~mm} \times 1.6 \mathrm{~mm}$. For a signal experiencing the maximum delay, the insertion loss was 11 dB. Phase measurements confirmed a maximum delay of $\sim 1.3$ ns for RF frequencies up to at least $\sim 25 \mathrm{GHz}$. With silicon nitride, a 4 bit discretely tunable delay with a maximum delay of $12.35 \mathrm{~ns}$ and $850 \mathrm{ps}$ resolution (step size) based on 5 cascaded $2 \times 2$ MZI switches and 4 waveguide paths was reported [48]. In this case, the longest delay path involved a waveguide length of $1.284 \mathrm{~m}$ in a spiral configuration with $<5$ $\mathrm{mm}$ bend radii and propagation losses of $\sim 1 \mathrm{~dB} / \mathrm{m}$. The device is compact, occupying a total chip size of $\sim 4.5 \times 8.5$ $\mathrm{cm}$.

One approach to minimize or reduce complexities with curvy or spiral/serpentine topologies in length variable OTTDLs is to develop an index variable OTTDL where true time delay control is realized through varying the group index/propagation velocity in the waveguides. Note that the ODL structure illustrated in Fig. 13(a) is not an OTTDL that provides time delays for signals at the same wavelength since the variable index/propagation velocity controls the relative wavelength dependent delay and not the true time delay. Recently, Gasulla and Capmany proposed to exploit the parallelism inherent in multicore fibers to implement a sampled true time delay line for MWP applications [61]: the use of a heterogeneous multicore fiber design provides a different delay for a given optical carrier at the output of each fiber (the time delay is controlled through designing a proper physical dimension and material doping concentration of each fiber core). By tailoring an independent dispersion profile per core, a differential/incremental delay between taps of a few $\mathrm{ps} / \mathrm{km}$ can be achieved [62]. We now describe an approach to realize an integrated version of this OTTDL concept.

\section{B. SWG waveguides for OTTDLs}

Recently, there has been growing interest in SWG structures for developing integrated optical components [63-66]. SWGs are formed by a periodic arrangement (with period $\Lambda$ ) of high refractive index material (e.g., silicon) with a thickness $a$ implanted into a low refractive index material (e.g., silica); the duty cycle of the SWG is defined as $D=a / \Lambda$, see Fig. 14(a). Efficient fiber-to-chip surface grating couplers in SOI based on crosswise operation, where light propagates perpendicularly to the subwavelength structure, have been reported $[65,66]$. Light can also propagate along the axis of the subwavelength structure, i.e., lengthwise operation, giving rise to the notion of SWG waveguiding.

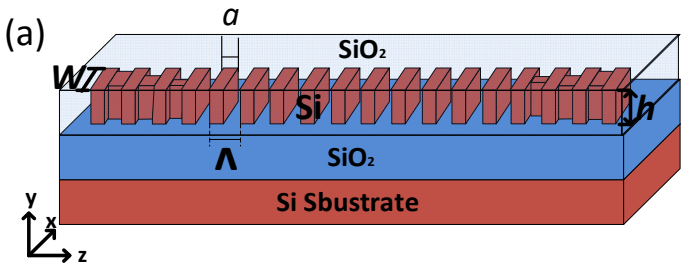

(b)

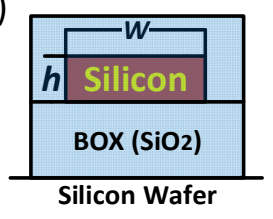

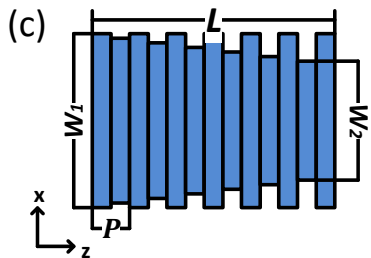

Fig. 14. (a) Schematic of SWG waveguide implemented in SOI, (b) waveguide cross-section, and (c) top view of SWG taper.

To create an SWG waveguide, finite transverse dimensions, e.g., a height $h$ and width $W$, are applied to the material of high refractive index. Light propagation along the axis of the subwavelength structure (periodic refractive index arrangement) can be understood in a similar manner to electron propagation in a periodic potential and can be described using Bloch waves. The operating regime of the SWG waveguide depends on the carrier frequency of the incident light signal. At low frequencies, the propagation constant increases with frequency as in a conventional waveguide, i.e., the subwavelength regime. At higher frequencies, Bragg reflection occurs and light is reflected back, i.e., the Bragg reflection regime. At even higher frequencies above this bandgap lies the radiation regime where the Bloch mode becomes leaky and light is scattered out of the waveguide, i.e., the radiation regime. We are particularly interested in the first operating regime-the subwavelength regime - where light perceives the periodic structure as an effective medium. In other words, the SWG waveguide can be modeled as a conventional nanowire waveguide having the same transverse dimensions and a uniform refractive (effective) index along the direction of propagation.

The important parameter of the SWG waveguide is the effective index $n$, which depends on the duty cycle $D[66]$ :

$n=\sqrt{D n_{1}^{2}+(1-D) n_{2}^{2}}$

where $n_{1}$ and $n_{2}$ are the effective refractive indices of the silicon and silica waveguides, respectively. The group index of the SWG waveguide $\left(n_{g}\right)$ can be calculated from the wellknown definition

$n_{g}=n+\omega \frac{d n}{d \omega}$,

where $\omega$ is the optical frequency. Substituting (6) in (7) gives

$n_{g}=\frac{n_{1} n_{g 1} D+n_{2} n_{g 2}(1-D)}{\sqrt{D n_{1}^{2}+(1-D) n_{2}^{2}}}$.

where $n_{\mathrm{g} 1}$ and $n_{\mathrm{g} 2}$ are the group indices of the silicon and silica waveguides as defined by (7).

In addition to SWG waveguides (with propagation losses of $\sim 2-3 \mathrm{~dB} / \mathrm{cm}$ ), a number of SWG waveguide devices have been demonstrated, such as directional and MMI couplers, 
waveguide crossings, bends, Bragg gratings, and ring resonators [63-70]. Moreover, the use of SWG waveguides to create a delay/path length mismatch in an MZI was demonstrated recently [71]. We build on this idea and take advantage of the ability to tune the effective index of an SWG waveguide through control of its duty cycle $D$ to realize an integrated index variable OTTDL [72].

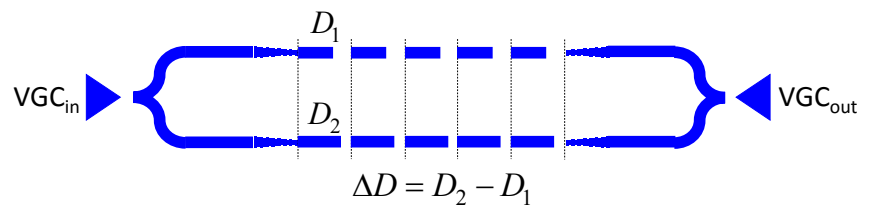

(a)

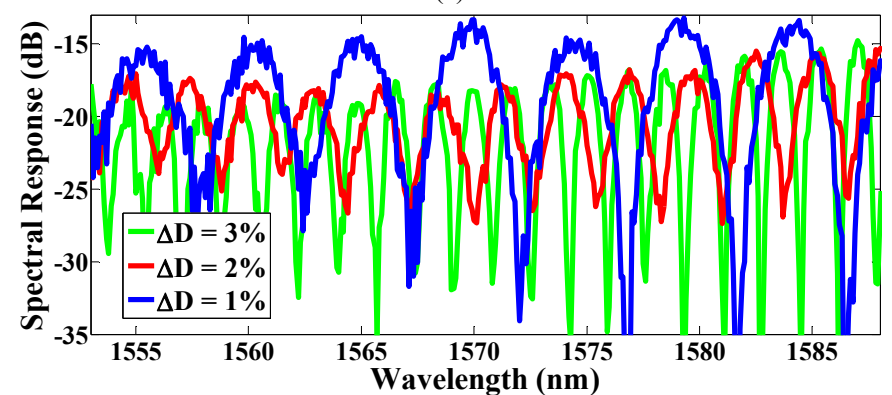

(b)

Fig. 15. (a) Schematic of MZI incorporating SWG waveguides with different duty cycles $D_{l}$ and $D_{2}$ in each arm. (b) Measured spectral responses of MZIs with three different values of $\Delta D$.

First, to investigate the minimum incremental delay that can be obtained with this approach, we fabricated MZIs incorporating SWG waveguides with different duty cycles in each arm $\left(\Delta D=D_{1}-D_{2}\right.$ where $D_{l}$ and $D_{2}$ are the duty cycles of the two SWG waveguides) as depicted in Fig. 15(a) [the same fabrication processes for developing the on-chip spectral shapers in SOI are used]. The measured FSR from each MZI can then be correlated with the optical delay/path length mismatch induced by $\Delta D$. The SWG waveguides have a cross-section of $h \times W=220 \mathrm{~nm} \times 500 \mathrm{~nm}$; as with the onchip spectral shapers, they sit on a $3 \mu \mathrm{m}$ BOX layer and are covered by a $2 \mu \mathrm{m}$ thick index-matched top oxide cladding. The SWG waveguides are coupled to conventional silicon nanowires using a taper which converts a mode propagating in a silicon nanowire waveguide into a Bloch mode that propagates in the SWG waveguide. The tapers are based on a linearly chirped waveguide grating implemented with a uniform grating period $P$ and where the waveguide width is narrowed linearly from $W_{1}$ to $W_{2}$ over a length Ltaper as illustrated in Fig. 14(c) [67]. In our devices, we use $P=200$ $\mathrm{nm}$ and the waveguide width is varied from $500 \mathrm{~nm}$ down to $200 \mathrm{~nm}$ over a length of $L_{\text {taper }}=15 \mu \mathrm{m}$. Two conventional Ybranches are used to form the MZI and VGCs optimized for TE polarization provide input and output coupling (the Ybranches and VGCs have the same design as those used for the on-chip spectral shapers). The SWG waveguides have a period $\Lambda=250 \mathrm{~nm}$ and the same length of $8 \mathrm{~mm}$. Fig. 15(b) shows the measured spectral responses of 3 MZIs with $\Delta D=$ $1 \%, 2 \%$, and $3 \%$; the FSRs are $\sim 5 \mathrm{~nm}, \sim 2.9 \mathrm{~nm}$, and $\sim 1.7$ $\mathrm{nm}$, respectively, corresponding to delay differences of $1.6 \mathrm{ps}$,
$2.8 \mathrm{ps}$, and $4.7 \mathrm{ps}$ between the MZI arms. These results demonstrate that an incremental time delay of a few picoseconds can be obtained readily between SWG waveguides of the same length using a small difference in duty cycle. Of course, the minimum incremental time delay will scale proportionally with the SWG waveguide length; as such, sub-picosecond delays are expected with shorter waveguides $(<8 \mathrm{~mm})$.

Next, we fabricated an array of SWG waveguides which can provide optical true time delay for microwave phase shifting, see Fig. 16(a). The SWG waveguides, tapers, and VGCs are the same as those used in the MZIs. By using a different duty cycle for each SWG waveguide, different optical paths among the waveguides which have the same length can be engineered to control the incremental time delay. The $4 \mathrm{SWG}$ waveguides are all $8 \mathrm{~mm}$ long and their effective indices are varied in $10 \%$ increments from $30 \%$ to $60 \%$ to provide an incremental time delay of $\sim 10 \mathrm{ps}$.

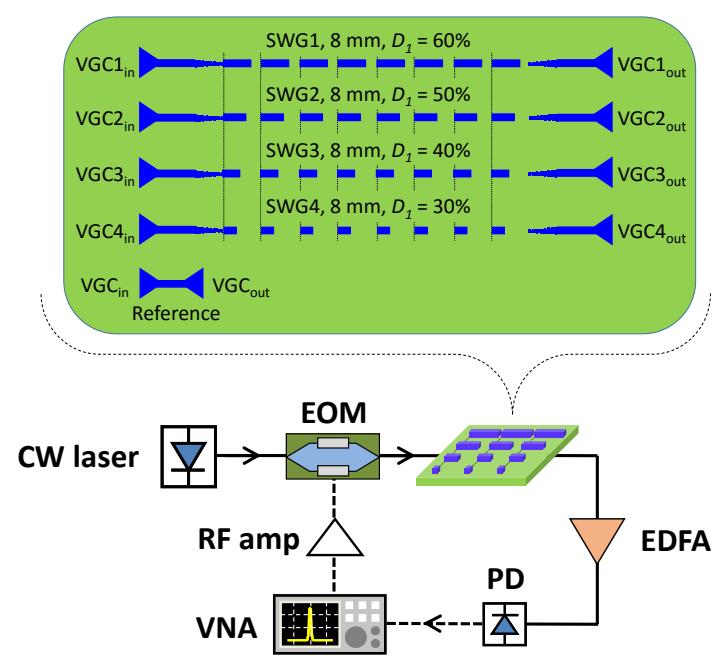

(a)

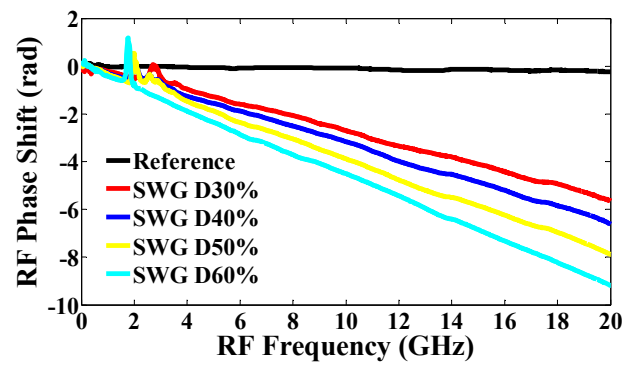

(b)

Fig. 16. (a) Schematic of OTTDL based on an array of 4 SWG waveguides and experimental setup for microwave phase shift measurements. (b) Measured RF phase shift vs. modulation frequency for an optical carrier at $1565 \mathrm{~nm}$.

A vector network analyzer (VNA) is used to measure the RF phase shift as a function of frequency as depicted in Fig. 16(a). Fig. 16(b) shows the measured RF phase shift as a function of RF frequency for the 4 waveguides at an optical carrier wavelength of $1565 \mathrm{~nm}$. The time delay of each waveguide can be obtained from the average slope of the phase shift. From the measurements, we estimate incremental time delays of $8.9 \mathrm{ps}, 10.7 \mathrm{ps}$, and $7.9 \mathrm{ps}$ between the SWG 
waveguides with $D_{1}=60 \%, D_{2}=50 \%, D_{3}=40 \%$, and $D_{4}=$ $30 \%$, respectively. The simulated effective and group indices of silicon and silica for our waveguide dimension at a wavelength of $\sim 1560 \mathrm{~nm}$ are $n_{1}=2.4, n_{\mathrm{g} 1}=4.4, n_{2}=1.45$, and $n_{\mathrm{g} 2}=1.47$. According to (8), the group indices of the SWG waveguides for $D=60 \%, 50 \%, 40 \%$, and $30 \%$ are $n_{\mathrm{g}}=3.47$, $3.2,2.91$, and 2.61 , respectively, and the corresponding incremental time delays are $7.1 \mathrm{ps}, 7.6 \mathrm{ps}$, and $8.2 \mathrm{ps}$, which are in fairly good agreement with the measured results.

\section{Discussion}

The total fiber-to-fiber loss for an SWG waveguide depends on several factors, including (1) coupling loss associated with the VGCs, (2) propagation loss in the SWG waveguide, and (3) loss due to mode mismatch between the nanowire waveguide and SWG waveguide. The mode mismatch is more pronounced for SWG waveguides with smaller values of duty cycle $D$ (it is also polarization dependent though this is not problematic in our case since the VGCs are optimized for operation on a single polarization only) [67]. While tapers can reduce mode mismatch, a taper loss still exists. Moreover, each duty cycle requires its own optimal taper design to reduce loss due to mode mismatch. In our proof-of-principle demonstration, we used the same taper design for all 4 SWG waveguides; as such, the total fiber-to-fiber loss between them is not the same and the loss is highest for the waveguide with $D=30 \%$ (it is several dB higher than for the waveguide with $D=60 \%$ ) [70]. More generally, if we choose to use the same taper design for simplicity, then minimizing the variation in fiber-to-fiber loss among the different SWG waveguides will require the use of smaller differences in duty cycle.

The minimum incremental time delay and the maximum time delay that can be obtained depend on the minimum and maximum differences in duty cycle that can be used. For example, with a difference in duty cycle of $\Delta D=1 \%$, an incremental time delay of $1.6 \mathrm{ps}$ can be obtained with $8 \mathrm{~mm}$ long SWG waveguides whereas with a difference in duty cycle of $\Delta D=30 \%$, the incremental delay increases to $27.5 \mathrm{ps}$. Reducing the incremental time delay can be achieved readily by reducing the length of the SWG waveguides and subpicosecond values are possible. On the other hand, increasing the maximum delay (and/or the maximum incremental delay) will require substantial increases in SWG waveguide length. It is possible to arrange the SWG waveguides in a serpentine manner to increase the total length while maintaining a compact size. For example, a $90^{\circ}$ bending loss of $\sim 1.5 \mathrm{~dB}$ was demonstrated for a bend radius of $10 \mu \mathrm{m}$; this loss was reduced to $\sim 1 \mathrm{~dB}$ when the bend radius was increased to 30 $\mu \mathrm{m}$. These losses can be reduced even further by utilizing trapezoidal silicon segments (as opposed to rectangular silicon segments) in the SWG waveguide bends [73]. As mentioned previously, the typical propagation losses in SWG waveguides are similar to those in silicon nanowire waveguides $[63,64]$. Thus, SWG waveguides should support not only small incremental delays (for high resolution) but also maximum delays of a few nanoseconds (similar to those reported in [49] which are based on silicon rib waveguides).

The index variable design allows the different taps of the OTTDL to be realized in a compact array of straight waveguides with identical lengths. Note that as with any OTTDL design, the SWG waveguides must be separated by a sufficient distance to avoid coupling (especially for a serpentine arrangement as SWG waveguides with the same duty cycle are placed in close proximity). The separation depends on duty cycle as stronger coupling has been observed for SWG waveguides with smaller values of $D$ [67].

It should be noted that to implement a discretely tunable OTTDL, $2 \times 2$ switches are required. SWG waveguide-based switches or modulators have yet to be demonstrated although all of the components exist for their implementation. On the other hand, it is possible to use a conventional silicon $2 \times 2$ MZI switches, i.e., the SWG waveguides are exploited only for developing the index variable delay elements whereas conventional silicon waveguides are used for other components in the tunable OTTDL device.

The features that an ODL are required to provide will have a strong influence on the implementation. For example, while SWG waveguides can support both large as well as small total or incremental delays, continuous tuning will be more challenging to achieve. On the other hand, wavelength variable ODLs, including the use of resonance enhancements based on coupled resonators $[7,50,51,53]$ or slow light effects in photonic crystal waveguides [52,57] or the dispersive properties of grating structures [55,56] (all in SOI) allow readily for continuous tuning. The tuning range, however, is typically limited from tens to $\sim 200 \mathrm{ps}$. While the delays can be obtained in very compact structures (e.g., in [52], photonic crystal waveguides with lengths from $280 \mu \mathrm{m}$ to $400 \mu \mathrm{m}$ are considered while in [57], the length is $1.5 \mathrm{~mm}$ ), the total insertion loss of the devices can be high (from $16 \mathrm{~dB}$ in [57] to $30 \mathrm{~dB}$ in [52]). It should be noted that a significant fraction of the loss tends to be associated with fiber coupling. For larger delays and discretely tunable delays, length variable and index variable ODLs will likely be preferred. At present, the fiberto-fiber loss of SWG waveguide devices (including tapers and VGCs) tends to be higher compared to tunable devices demonstrated in silicon nitride [48] and SOI [49]. Further improvements in SWG waveguide device design as well as fabrication are required to make them more competitive with SOI structures.

\section{SUMMARY AND OUTLOOK}

We have reviewed recent developments of integrated microwave photonic subsystems in SOI for generating chirped microwave waveforms and providing optical true time delay. The design and realization of on-chip spectral shapers based on BGs (particularly a DFPC, a Michelson interferometer incorporating identical chirped BGs, and a Sagnac loop incorporating a single chirped $\mathrm{BG}$ ) to enable waveform generation via spectral shaping and WTM was discussed. These on-chip spectral shapers can be used to synthesize chirped microwave waveforms with an RF chirp and TBWPs that are comparable with all-fiber or free-space approaches; moreover, they outperform those based on MRRs. While this passive spectral shaping approach cannot generate similar TBWPs to those available from heterodyne beating, the valueadded with on-chip spectral shapers may lie in the tunability (e.g., using the plasma dispersion effect in pn junction 
waveguides), compactness, and the feasibility to develop parallel structures for simultaneous generation of multiple chirped waveforms.

We also described how an index variable OTTDL can be implemented using SWG waveguides. In particular, the ability to tune the group index of an SWG waveguide by controlling its duty cycle provides a straightforward mechanism to create different propagation delays between waveguides of the same length. A difference in duty cycle of only $1 \%$ (which can be readily obtained with ebeam processing) results in an incremental delay of $1.6 \mathrm{ps}$ for waveguides $8 \mathrm{~mm}$ in length; sub-picosecond incremental delays are thus possible with shorter waveguides. To increase the incremental delay and/or achieve larger total delays, a greater different in duty cycle is required. However, using large differences will result in greater variations in fiber-tofiber loss, unless the tapers are specifically optimized for each SWG waveguide. Alternatively, longer SWG waveguides can be employed. While the propagation loss in SWG waveguides in SOI is similar to that in silicon nanowire waveguides, they are significantly higher than that available with silicon nitride waveguides. As such, for applications requiring very long delays, e.g., exceeding several nanoseconds, silicon nitride may be the material platform of choice. Since SWG waveguides are based on the same technology and waveguide design as silicon nanowires, they are compatible with existing silicon photonic devices, e.g., switches, modulators, etc. As such, SWG waveguide enhance the available component toolbox for developing integrated microwave photonic subsystems in SOI. Finally, SWG waveguide crossings with low loss $(0.1-0.2 \mathrm{~dB})$ and low crosstalk $(<-40 \mathrm{~dB})$ have been demonstrated [64]. As such, they offer have the promise for very high density integration.

The technological developments described in this paper, along with others in silicon photonics such as high performance modulators and detectors, point to the feasibility of more complex integrated microwave photonic (sub-) systems that can provide increased functionality and ultimately performance.

\section{ACKNOWLEDGEMENTS}

The on-chip spectral shapers and SWG waveguide devices were fabricated by R. Bojko at the University of Washington Nanofabrication Facility (WNF), a member of the NSF National Nanotechnology Infrastructure Network. I thank Dr. J. Wang, M. Ma, Dr. R. Ashrafi (McGill University), R. Adams (CEGEP Vanier College), Prof. I Glesk (University of Strathclyde), Dr. I. Gasulla, and Prof. J. Capmany (iTEAM, Universitat Politècnica de València) for their contributions to this work.

\section{REFERENCES}

[1] A. J. Seeds and K. J. Williams, "Microwave photonics," IEEE/OSA J. Lightw. Technol., vol. 24, no. 12, pp. 4628-4641, Dec 2006.

[2] J. Capmany and D. Novak, "Microwave photonics combines two worlds," Nat. Photon., vol. 1, pp. 319-330, Jun 2007.

[3] S. Iezekiel, M. Burla, J. Klamkin, D. Marpaung, and J. Capmany, "RF engineering meets optoelectronics," IEEE Microw. Magazine, vol. 16, no. 8, pp. 28-45, Sep 2015.
[4] J. Yao, "Microwave photonics," IEEE/OSA J. Lightw. Technol., vol. 27, no. 3, pp. 314-335, Feb 2009.

[5] S. J. Ben Yoo, R. P. Scott, D. J. Geisler, N. K. Fontaine, and F. M. Soares, "Terahertz information and signal processing by RF-photonics," IEEE Trans. on Terahertz Sci. and Technol., vol. 2, no. 2, pp. 167-176, Mar 2012.

[6] D. Marpaung, C. Roeloffzen, R. Heideman, A. Leinse, S. Sales, and J. Capmany, "Integrated microwave photonics," Lasers \& Photon. Rev., vol. 7, no. 4, pp. 506-538, Jul 2013.

[7] C. G. H. Roeloffzen, L. Zhuang, C. Taddei, A. Leinse, R. G. Heideman, P. W. L. van Dijk, R. M. Oldenbeuving, D. A. I. Marpaung, M. Burla, and K.-J. Boller, "Silicon nitride microwave photonic circuits," Opt. Express, vol. 21, no. 19, pp. 22937-22961, Sep 2013.

[8] M. Burla, L. R. Cortés, M. Li, X. Wang, L. Chrostowski, and J. Azaña, "Integrated waveguide Bragg gratings for microwave photonics signal processing," Opt. Express, vol. 21, no. 21, pp. 25120-25147, Oct 2013.

[9] D. Marpaung, M. Pagani, B. Morrison, and B. J. Eggleton, "Nonlinear integrated microwave photonics," IEEE/OSA J. Lightw. Technol., vol. 32, no. 20, pp. 3421-3427, Oct 2014.

[10] W. Zhang and J. Yao, "Silicon-based integrated microwave photonics," IEEE J. Quantum Electron., vol. 52, no. 1, 0600412, Jan 2016.

[11] J. Yao, "Photonic generation of microwave arbitrary waveforms," Opt. Commun., vol. 284, no. 15, pp. 3723-3736, Jul 2011.

[12] A. M. Weiner, "Optical pulse shaping: A tutorial review," Opt. Commun., vol. 284, no. 15, pp. 3669-3692, Jul 2011.

[13] J. Chou, Y. Han, and B. Jalali, "Adaptive RF-photonic arbitrary waveform generation," IEEE Photon. Technol. Lett., vol. 15, no. 4, pp. 581-583, Apr 2003.

[14] I. S. Lin, J. D. McKinney, and A. M. Weiner, "Photonic synthesis of broadband microwave arbitrary waveforms applicable to ultra-wideband communication," IEEE Microw. and Wireless Compon. Lett., vol. 15, no. 4, pp. 226-228, Apr 2005.

[15] V. Torres-Company, J. Lancis, and P. Andrés, "Arbitrary waveform generator based on all-incoherent pulse shaping," IEEE Photon. Technol. Lett., vol. 18, no. 24, pp. 2626-2628, Dec 2006.

[16] H. Chi, F. Zeng, and J. Yao, "Photonic generation of microwave signals based on pulse shaping," IEEE Photon. Technol. Lett., vol. 19, no. 9, pp. 668-670, May 2007.

[17] H. Chi and J. Yao, "Chirped RF pulse generation based on optical spectral shaping and wavelength-to-time mapping using a nonlinearly chirped fiber Bragg grating," IEEE/OSA J. Lightw. Technol., vol. 26, no. 10, pp. 1282-1287, May 2008.

[18] L. R. Chen, "Photonic generation of chirped microwave and millimeter wave pulses based on optical spectral shaping and wavelength-to-time mapping in silicon photonics," Opt. Commun., in press, 2016.

[19] S. Doucet, R. Slavík, and S. LaRochelle, "High-finesse large band Fabry-Pérot fiber filter with superimposed chirped Bragg gratings," IET Electron. Lett., vol. 38, no. 9, pp. 402-403, Apr 2002.

[20] R. Slavík, S. Doucet, and S. LaRochelle, "High-performance all-fiber Fabry-Pérot filters with superimposed chirped Bragg gratings," IEEE/OSA J. Lightw. Technol., vol. 21, no. 4, pp. 1059-1065, Apr 2003.

[21] C. Wang and J. Yao, "Photonic generation of chirped microwave pulses using superimposed chirped fiber Bragg gratings," IEEE Photon. Technol. Lett., vol. 20, no. 11, pp. 882-884, Jun 2008.

[22] M. Ma and L. R. Chen, "Generating chirped microwave pulses using an integrated distributed Fabry-Pérot cavity in silicon-on-insulator," IEEE Photon. J., vol. 7, no. 2, 5500706, Apr 2015.

[23] L. Chrostowski and M. Hochberg, Silicon Photonics Design: From Devices to Systems, Cambridge University Press, 2015.

[24] W. Wang, W. Shi, R. Vafei, N. A. F. Jaeger, and L. Chrostowski, "Uniform and sampled Bragg gratings in SOI strip waveguides with sidewall corrugations," IEEE Photon. Technol. Lett., vol. 23, no. 5, pp. 290-292, Mar 2011.

[25] I. Giuntoni, D. Stolarek, D. I. Kroushkov, J. Bruns, L. Zimmermann, B. Tillack, and K. Petermann, "Continuously tunable delay line based on SOI tapered Bragg gratings," Opt. Express, vol. 20, no. 10,pp. 1124111246, May 2012.

[26] R. Kashyap, Fiber Bragg Gratings, Academic Press, 1999.

[27] W. Zhang and J. Yao, "Photonic generation of linearly chirped microwave waveforms using a silicon-based on-chip spectral shaper incorporating two linearly chirped waveguide Bragg gratings," 
IEEE/OSA J. Lightw. Technol., vol. 33, no. 24, pp. 5047-5054, Dec. 2015.

[28] X. Shu, L. Yu, D. Zhao, L. Zhang, K. Sugden, and I. Bennion, "Transmission characteristics of Sagnac interferometers based on fiber Bragg gratings," J. Opt. Soc. Am. B, vol. 19, no. 11, pp. 2770-2780, Nov 2002.

[29] C. Wang and J. Yao, "Chirped microwave pulse generation based on optical spectral shaping and wavelength-to-time mapping using a Sagnac loop mirror incorporating a chirped fiber Bragg grating," IEEE/OSA J. Lightw. Technol., vol. 27, no. 16, pp. 3336-3341, Aug 2009.

[30] J. Wang, R. Ashrafi, M. Rochette, and L. R. Chen, "Chirped microwave pulse generation using an integrated silicon photonic spectral shaper," IEEE Photon. Technol. Lett., vol. 27, no. 17, pp. 1876-1879, Sep 2015.

[31] M. H. Khan, H. Shen, Y. Xuan, L. Zhao, S. Xiao, D. E. Leaird, A. M. Weiner, and M. Qi, "Ultrabroadbandwidth arbitrary radiofrequency waveform generation with a silicon photonic chip-based spectral shaper," Nat. Photon., vol. 4, pp. 117-122, Feb 2010.

[32] R. Ashrafi, Y. Park, and J. Azaña, "Fiber-based photonic generation of high-frequency microwave pulses with reconfigurable linear chirp control," IEEE Trans. on Microw. Theory and Techn., vol. 58, no. 11, pp. 3312-3319, Nov 2010.

[33] A. Rashidinejad and A. M. Weiner, "Photonic radio-frequency arbitrary waveform generation with maximal time-bandwidth product capability," IEEE/OSA J. Lightw. Technol., vol. 32, no. 20, pp. 3383-3393, Oct 2014.

[34] Y. Li, A. Rashidinejad, J.-M. Wun, D. E. Leaird, J.-W. Shi, and A. M. Weiner, "Photonic generation of W-band arbitrary waveforms with high time-bandwidth products enabling $3.9 \mathrm{~mm}$ range resolution," Optica, vol. 1, no. 6, pp. 446-454, Dec 2014.

[35] J.-W. Shi, F.-M. Kuo, N.-W. Chen, S. Y. Set, C.-B. Huang, and J. E. Bowers, "Photonic generation and wireless transmission of linearly/nonlinearly continuously chirped millimeter-wave waveforms with high time-bandwidth product at W-band," IEEE Photon. J., vol. 4, no. 1, pp. 215-223, Feb 2012.

[36] W. Zhang and J. P. Yao, "Silicon-based on-chip electrically-tunable spectral shaper for continuously tunable linearly chirped microwave waveform generation," IEEE/OSA J. Lightw. Technol., to appear, 2016.

[37] K. Sasaki, F. Ohno, A. Motegi, and T. Baba, "Arrayed waveguide grating of $70 \times 60 \mu \mathrm{m}^{2}$ size based on Si photonic wire waveguides," IET Electron. Lett., vol. 41, no. 14, pp. 801-802, Jul 2005.

[38] W. Bogaerts, P. Dumon, D. Van Thourhout, D. Taillaert, P. Jaenen, J. Wouters, S. Becks, V. Wiaux, and R. G. Baets, "Compact wavelengthselective functions in silicon-on-insulator photonic wires," IEEE J. Sel. Top. in Quantum Electron., vol. 12, no. 6, pp. 1394-1401, Nov/Dec 2006.

[39] J. Capmany, P. Muñoz, S. Sales, D. Pastor, B. Ortega, and A. Martinez, "Arrayed waveguide Sagnac interferometer," Opt. Lett., vol. 28, no. 3, pp. 197-199, Feb 2003.

[40] J. Capmany, J. Mora, I. Gasulla, J. Sancho, J. Lloret, and S. Sales, "Microwave photonic signal processing," IEEE/OSA J. Lightw. Technol., vol. 31, no. 4, pp. 571-586, Feb. 2013.

[41] R. Minasian, E. W. H. Chan, and X. Yi, "Microwave photonic signal processing," Opt. Express, vol. 21, no. 19, pp. 22918-22936, Sep 2013.

[42] I. Frigyes and A. J. Seeds, "Optically generated true-time delay in phased-array antennas," IEEE Trans. Microw. Theory and Techn., vol. 43, no. 9, pp. 2378-2386, Sep 1995.

[43] W. Ng, A. A. Walston, G. L. Tangonan, J. J. Lee, I. L. Newberg, and N. Bernstein, "The first demonstration of an optically steered microwave phased array antenna using true-time-delay," IEEE/OSA J. Lightw. Technol., vol. 9, no. 9, pp. 1124-1131, Sep 1991.

[44] V. Kaman, X. Zheng, R. J. Helkey, C. Pusarla, and J. E. Bowers, "A $32-$ element 8-Bit photonic true-time-delay system based on a $288 \times 2883$ D MEMS optical switch," IEEE Photon. Technol. Lett., vol. 15, no. 6, pp. 849-851, Jun 2003.

[45] J. D. Shin, B. S. Lee, and B. G. Kim, "Optical true time-delay feeder for $\mathrm{X}$-band phased array antennas composed of $2 \times 2$ optical MEMS switches and fiber delay lines," IEEE Photon. Technol. Lett., vol. 16, no. 5, pp. 1364-1366, May 2004.

[46] S. Yegnanarayanan, P. Trinh, F. Coppinger, and B. Jalali, "Compact silicon-based integrated optic time delays," IEEE Photon. Technol. Lett., vol. 9, no. 5, pp. 634-635, May 1997.
[47] H. Lee, T. Chen, J. Li, O. Painter, and K. J. Vahala, "Ultra-low-loss optical delay line on a silicon chip," Nat. Commun., vol. 3, 867, May 2012.

[48] R. L. Moreira, J. Garcia, W. Li, J. Bauters, J. S. Barton, M. J. R. Heck, J. E. Bowers, and D. J. Blumenthal, "Integrated ultra-low-loss 4-bit tunable delay for broadband phased array antenna applications," IEEE Photon. Technol. Lett., vol. 25, no. 12, pp. 1165-1168, Jun 2013.

[49] J. Xie, L. Zhou, Z. Li, J. Wang, and J. Chen, "Seven-bit reconfigurable optical true time delay line based on silicon integration," Opt. Express, vol. 22, no. 19, pp. 22707-22715, Sep 2014.

[50] M. S. Rasras, C. K. Madsen, M. A. Cappuzzo, E. Chen, L. T. Gomez, E. J. Laskowski, A. Griffin, A. Wong-Foy, A. Gasparyan, A. Kasper, J. Le Grange, and S. S. Patel, "Integrated resonance-enhanced variable optical delay lines," IEEE Photon. Technol. Lett., vol. 17, no. 4, pp. 834-836, Apr 2005.

[51] J. Cardenas, M. A. Foster, N. Sherwood-Droz, C. B. Poitras, H. L. R. Lira, B. Zhang, A. L. Gaeta, J. B. Khurgin, P. Morton, and M. Lipson, "Wide-bandwidth continuously tunable optical delay line using silicon microring resonators," Opt. Express, vol. 18, no. 25, pp. 26525-26534, Dec 2010

[52] J. Adachi, N. Ishikura, H. Sasaki, and T. Baba, "Wide range tuning of slow light pulse in SOI photonic crystal coupled waveguide via folded chirping," IEEE J. Sel. Top. in Quantum Electron., vol. 16, no. 1, pp. 192-199, Jan/Feb 2010.

[53] A. Melloni, A. Canciamilla, C. Ferrari, F. Morichetti, L. O'Faolain, T. F. Krauss, R. De La Rue, A. Samarelli, and M. Sorel, "Tunable delay lines in silicon photonics: coupled resonators and photonic crystals: a comparison," IEEE Photon. J., vol. 2, no. 2, pp. 181-194, Apr 2010.

[54] M. Burla, D. Marpaung, L. Zhuang, C. Roeloffzen, M. Rezaul Khan, A. Leinse, M. Hoekman, and René Heideman, "On-chip CMOS compatible reconfigurable optical delay line with separate carrier tuning for microwave photonic signal processing," Opt. Express, vol. 19, no. 22, pp. 21475-21484, Oct 2011.

[55] S. Khan, and S. Fathpour, "Complementary apodized grating waveguides for tunable optical delay lines," Opt. Express, vol. 20, no. 18, pp. 19859-19867, Aug 2013.

[56] S. Khan and S. Fathpour, "Demonstration of complementary apodized cascaded grating waveguides for tunable optical delay lines," Opt. Lett. vol. 38, no. 19, pp. 3914-3917, Oct 2013.

[57] J. Sancho, J. Bourderionnet, J. Lloret, S. Combrié, I. Gasulla, S. Xavier, S. Sales, P. Colman, G. Lehoucq, D. Dolfi, J. Capmany, and A. D. Rossi, "Integrable microwave filter based on a photonic crystal delay line," Nat. Commun., vol. 3, 1075, Sep 2012.

[58] M. Spasojevic and L. R. Chen, "Discretely tunable optical delay lines using serial and step-chirped sidewall Bragg gratings in SOI," IET Electron. Lett., vol. 49, no. 9, pp. 608-610, Apr 2013.

[59] J. Xie, L. Zhou, Z. Zou, J. Wang, X. Li, and J. Chen, "Continuously tunable reflective-type optical delay lines using microring resonators," Opt. Express, vol. 22, no. 1, pp. 817-823, Jan 2014.

[60] G. Lenz, B. J. Eggleton, C. K. Madsen, and R. E. Slusher, "Optical delay lines based on optical filters," IEEE J. Quantum Electron., vol. 37, no. 4, pp. 525-532, Apr 2001.

[61] I. Gasulla and J. Capmany, "Microwave photonics applications of multicore fibers," IEEE Photon. J., vol. 4, no. 3, pp. 877-888, Jun 2012.

[62] S. Garcia and I. Gasulla, "Design of heterogeneous multicore fibers as sampled true-time delay lines," Opt. Lett., vol. 40, no. 4, pp. 621-624, Feb 2015.

[63] P. J. Bock, P. Cheben, J. H. Schmid, J. Lapointe, A. Delâge, S. Janz, G. C. Aers, D.-X. Xu, A. Densmore, and T. J. Hall, "Subwavelength grating periodic structures in silicon-on-insulator: a new type of microphotonic waveguide," Opt. Express, vol. 18, no. 19, pp. 20251-20262, Sep 2010.

[64] J.-H. Schmid, P. Cheben, P. J. Bock, R. Halir, J. Lapointe, S. Janz, A. Delâge, A. Densmore, J.-M. Fédeli, T. J. Hall, B. Lamontagne, R. Ma, I. Molina-Fernández, and D.-X. Xu, "Refractive index engineering with subwavelength gratings in silicon microphotonic waveguides," IEEE Photon. J., vol. 3, no. 3, pp. 597-607, Jun 2011.

[65] R. Halir, A. Ortega-Moñux, J. H. Schmid, C. Alonso-Ramos, J. Lapointe, D.-X. Xu, J. G. Wangüemert-Pérez, I. Molina-Fernández, and S. Janz, "Recent advances in silicon waveguide devices using subwavelength gratings," IEEE J. of Sel. Top. in Quantum Electron, vol. 20, no. 4, pp. 279-291, Jul/Aug 2014.

[66] R. Halir, P. J. Bock, P. Cheben, A. Ortega-Moñux, C. Alonso-Ramos, J. H. Schmid, J. Lapointe, D.-X. Xu, J. G. Wangüemert-Pérez, Í. Molina- 
Fernández, and S. Janz, "Waveguide sub-wavelength structures: a review of principles and applications," Lasers \& Photon. Rev., vol. 9, no. 1, pp. 25-49, Jan 2015.

[67] V. Donzella, A. Sherwali, J. Flueckiger, S. T. Fard, S. M. Grist, and L. Chrostowski, "Sub-wavelength grating components for integrated optics applications on SOI chips," Opt. Express, vol. 22, no. 17, pp. 2103721050, Aug 2014.

[68] J. Wang, I. Glesk, and L. R. Chen, "Subwavelength grating filtering devices," Opt. Express, vol. 22, no. 13, pp. 15335-15345, Jun 2014.

[69] J. Wang, I. Glesk, and L. R. Chen, "Subwavelength grating Bragg grating filters in silicon-on-insulator," IET Electron. Lett., vol. 51, no. 9, pp. 712-713, Apr 2015.

[70] J. Wang, I. Glesk, and L. R. Chen, "Subwavelength grating devices in silicon photonics," Sci. Bulletin, in press, 2016.

[71] I. Glesk, P. J. Bock, P. Cheben, J. H. Schmid, J. Lapointe, and S. Janz, "All-optical switching using nonlinear subwavelength Mach-Zehnder on silicon," Opt. Express, vol. 19, no. 15, pp. 14031-14039, Jul 2011.

[72] R. Ashrafi, J. Wang, R. Adams, I. Glesk, I. Gasulla, J. Capmany, and L. R. Chen, "Subwavelength grating enabled on-chip ultra-compact optical true time delay line," Scientific Rep., vol. 6, 30325, Jul 2016.

[73] Z. Wang, X. Xu, D. Fan, Y. Wang, H. Subbaraman, and R. T. Chen, "Geometrical tuning art for entirely subwavelength grating waveguide based integrated photonic circuits," Scientific Rep., vol. 6, 24106, May 2016.
Lawrence R. Chen (S'95-SM'05) received the B.Eng. degree in electrical engineering and mathematics from McGill University, Montreal, QC, Canada, in 1995 and the M.A.Sc. and Ph.D. degrees in electrical and computer engineering in 1997 and 2000, respectively. Since 2000, he has been with the Department of Electrical and Computer Engineering at McGill University. His research interests are in optical communications, fiber and integrated optics, and microwave photonics (in particular, active and passive devices in silicon photonics for optical and microwave signal processing) as well as engineering education and teaching pedagogy.

Dr. Chen is the Editor-in-Chief for the IEEE Photonics Society Newsletter, an Editor for Optics Communications, and an Associate Editor for IET Electronics Letters. 